\title{
Genetic Testing for Every Breast Cancer Patient: Would it be Real?
}

\section{Mert Tanal* and Bulent Citgez}

Department of General Surgery, Istanbul Sisli Hamidiye Etfal Research and Training Hospital, University of Health Sciences, Turkey

*Corresponding author: Mert Tanal, General surgeon, University of Health Sciences, Sisli Hamidiye Etfal Research and Training Hospital, Etfal Street No:3, Sisli Istanbul, Turkey,

\begin{tabular}{|c|}
\hline Mini Review \\
Volume 4 Issue 1 \\
Received Date: February 02, 2020 \\
Published Date: February 11, 2020 \\
DOI: $10.23880 /$ ijsst-16000141 \\
\hline
\end{tabular}
Tel:+905337215062; Email: merttanal@yahoo.com

\section{Background}

Approximately 10 to 15 percent of breast cancers can be attributed to a hereditary genetic mutation. In fact, since guidelines and tests are not well known today, only one out of five breast cancer patients who follow current guidelines are referred for genetic testing. Likewise, while most of the guides tends to give importance to details, it makes the doctors difficult to follow the management scheme, and as a result, the patient's examinations may not be completed. Our aim is to provide a brief, memorable summary for the current genetic tests and indications.

\section{The Importance of Genetic Mutation Detection}

Identifying harmful genetic mutations in breast cancer susceptibility genes can have many important effects for the breast cancer patient. These include a change in the patient's cancer management, advanced management strategies for recurrent breast cancer, management and prevention of other cancers, and gradual genetic tests for other individuals at risk.

The American Association of Breast Surgery (ASBrS) published a statement about tests for hereditary breast cancer syndromes in February 2019. The most important note drawn from these guidelines is that genetic testing should be available for germline mutations for each breast cancer patient; however, despite the consensus, the difficulties of genetic testing should be considered and appropriate strategies should be identified.

In the light of recent studies and clinical facts, there are five important points from ASBrS guidelines, in which, prioritization of patients for genetic tests are reflected.

\section{Five important points from ASBrS Consensus:}

1) Doctors experienced in genetic testing can discuss and organize genetic testing for patients[1].

2) It may be helpful to refer complicated patients to certified genetic counselors[1].

3) A germline mutation genetic test should be offered to both newly diagnosed and already-treated patients.

4) Patients, who have been genetically tested before, can also benefit from the updated test.

Especially patients tested before 2014, would not be tested for large genomic rearrangements of BRCA 1 and 2, as well as not tested for other potentially relevant genes such as PALB2 $[1,2]$.

5) Genetic testing should be performed for patients who are not diagnosed as cancer but currently meet the NCCN guidelines.

When it is not feasible to test the affected patient first, or when the patient is lost, the unaffected patient can be tested both with the routine gene-panel, with the multi-gene panel if the family history is incomplete (i.e., uncertainty about the type of cancer in the family history).

Patients and health care professionals should know that the genetic testing is only a tool to assess the risk of the cancer. Having a negative test result does not mean that the patient is not at increased risk for developing breast cancer, whereas a positive genetic test result especially in some hereditary breast cancer genes, such as CHEK2 variants, have low penetrance, which means that the patient does not have to develop breast cancer $[3,4]$.

In addition to these, there is a definition of "variant of uncertain significance". If the patient does not have the clinical finding and family history, and yet, there is a genetic testing result of "BRCA1 variant of uncertain significance", these patients should not be recommended for a change in the management. These genetic tests are not defining the management procedure of the patients, but only help the health care professionals to decide. With the alterations in the genetic testing and new developments, probably the gray 


\section{International Journal of Surgery \& Surgical Techniques}

zones of uncertainty will become clearer in near future [5].

\section{Results}

ASBrS recently published consensus guidelines recommending genetic testing for all breast cancer patients. This exhibits a separate attitude from other guidelines, such as the NCCN guideline. However, the difficulties of the application of this guideline are also discussed and also makes recommendations for over-diagnose and overtreatment situations such as prophylactic mastectomy for some gene mutation carriers. Wide genetic testing practices should be considered, studies should be focused on foreseeing the benefits of treatment on patient basis and it should be considered that genetic testing will turn into a standard examination in the treatment of breast cancer in the upcoming years.

\section{References}

1. Plichta J, Sebastian M, Hughes K, Smith LA, Menendez CS, et al. (2019) Germline genetic testing: What the breast surgeon needs to know. Ann of Surg Oncol26(7): 2184-2190.

2. Kapoor NS, Curcio LD, Blakemore CA, Bremner AK, McFarland RE, et al. (2015) Multigene panel testing detects equal rates of pathogenic BRCA $1 / 2$ pathogenic variants and has a higher diagnostic yield compared to limited BRCA $1 / 2$ analysis alone in patients at risk for hereditary breast cancer. Ann Surg Oncol 22(10): 32823288.

3. Schmidt MK, Hogervorst F, van Hier R, Cornelissen S, Broeks A, et al. (2016) Age and tumor subtype specific breast cancer risk estimates for CHEK2*1100delC carriers. J Clin Oncol 34(23): 2750-2760.

4. Milne RL (2009) Variants in the ATM gene and breast cancer susceptibility. Genome Med 1(1): 12.

5. LaDuca H, Stuenkel AJ, Dolinsky JS, Keiles S, Tandy S, et al. (2014) Utilization of multigene panels in hereditary cancer predisposition testing: analysis of more than 2000 patients. Genet Med 16(11): 830-837. 